\title{
Training Community Health Workers to Scale-Up HIV Care in Rural Lesotho: Implementation Lessons from the Field
}

\author{
Jonas Rigodon $^{1}$, Keith Joseph ${ }^{1}$, Salmaan Keshavjee ${ }^{2}$, Corrado Cancedda ${ }^{2}$, Mona Haidar ${ }^{3}$, \\ Nicolas Lesia ${ }^{1}$, Limpho Ramangoaela ${ }^{1}$, Jennifer Furin ${ }^{4,5 *}$ \\ ${ }^{1}$ Partners in Health, Boston, USA; ${ }^{2}$ Harvard Medical School, Boston, USA; ${ }^{3}$ Lebanese American University, Beirut, Lebanon; ${ }^{4}$ Case \\ Western Reserve University, TB Research Unit, Cleveland, USA; ${ }^{5}$ School of Medicine, Case Western Reserve University, Cleveland, \\ USA. \\ Email: *jenniferfurin@gmail.com
}

Received July $2^{\text {nd }}, 2012$; revised August $2^{\text {nd }}, 2012$; accepted August $10^{\text {th }}, 2012$

\begin{abstract}
Community health workers (CHWs) have long played an important role in the management of complex health problems, especially in resource-poor settings. Although there is a large literature regarding the use of CHWs around the world, there is little detail about how these workers are selected, trained and utilized in the field. Lesotho has one of the highest rates of HIV in the world, with an estimated $25 \%$ of the general population infected with the disease; at the same time, there is a significant health human resources shortage in Lesotho with an estimated $60 \%$ of health posts left vacant. Community health workers have the potential to play a major role in HIV treatment scale-up in the country, and in 2006, a CWH-based project called "The Rural Initiative" was started in the remote mountain regions of the country. More than 1000 CHWs were trained and employed through this program between June 2006 and December 2008. This paper will review the CHW program in detail, with a focus on recruitment, training, ongoing supervision and support, and the larger public health implications of the CHW program in Lesotho. It is hoped this program can serve as a practical model for other programs working with or in need of CHWs.
\end{abstract}

Keywords: Lesotho; Community Health Workers; HIV

\section{Introduction}

Community health workers (CHWs) are a group of health para-professional that have been used to treat myriad disease problems for decades [1]. Beginning with the "barefoot doctors" in rural China [2], CHW-based programs have expanded globally and there is an estimated 1.3 million such workers used in health programs around the world [3]. Although programs use varying definitions of CHWs, they are generally defined as individuals with little formal training in health but with great expertise in knowledge of the population and regions in which they work [4] When provided with program-specific training and support, these individuals play a key role in supporting and implementing health programs in the field [5]. Their activities can include disease screening, disease prevention health education, adherence support, and community mobilization [6-8].

CHWs are involved in the management of multiple diseases ranging from diabetes [9] to mental health [10]

${ }^{*}$ Corresponding author. to TB $[11,12]$. One health area in which CHWs have been invaluable is in the prevention, support and treatment of HIV [13-15]. CWHs have been a cornerstone in scaling up HIV prevention and treatment all over the world; they have been especially important in the high-burden HIV countries of southern Africa where there are also extreme shortages of trained health professionals [16-18].

Lesotho is one such country, with an estimated HIV seroprevalence of $25 \%$ [19]. Health care worker shortages are glaring in this country of two million people, with an estimated 80 physicians in the country and $60 \%$ of nursing posts vacant [20]. This health care worker shortage is acutely felt in the rural regions of the country, where health posts and clinics are often un-staffed and little is offered to these populations in terms of primary care let alone preventing testing and treating HIV [21].

In June of 2006, a program called the Rural Health initiative (RHI) was launched in the mountains of Lesotho to provide comprehensive HIV testing and treatment services to the populations there [22]. CWHs were an integral part of this program and were recruited and trained 
from the local populations as soon as the program began. Although much has been written about the use of CHWs in managing HIV, there is little scholarly literature about how CWHs are selected, trained, and supported in their work. This paper fills that gap by providing such information on CWHs at one clinic participating in the RHI.

\section{Setting}

In 2006, a joint program-involving The Ministry of Health and Social Welfare of Lesotho (MOHSW), the US-based NGO Partners In Health, The Division of Global Health Equity of Brigham and Women's Hospital, Boston, The Clinton HIV/AIDS Initiative, and Irish Aid-called the Rural Health Initiative (RHI) was launched [23]. The goal of this program was to increase access to HIV care and treatment in rural Lesotho. The program focused on seven mountain clinics and an intensive effort was made to introduce HIV services into settings of primary care [24]. As part of this program, CHWs were recruited and trained to assist in treatment adherence, active case finding, community mobilization, and patient education. The CHW component of this program was based on successful CWH program implemented in Haiti, Peru and Rwanda by Partners in Health [25]. Since starting in June, 2006, the RHI has provided primary care services to hundreds of thousands of individuals. Between June, 2006 and December, 2008, 13,887 individuals underwent HIV testing through the program. The RHI enrolled 4521 patients in HIV care and started 2354 on ART during this period of time. More than $85 \%$ of patients started in treatment remained on therapy as of December 31, 2008 [26]. A total of 1012 CHWs were trained as part of this work.

One clinic in the Mohale's Hoek region called "Nohana clinic" was the first site to begin implementing the RHI model. It was selected for logistical reasons and because there was a great deal of political and community support in the region. Because it was the first and most active clinic, the CHW program at Nohana clinic is the focus of this report.

\section{Methods}

This report is based on field observations that occurred over a 30 months time period by the authors using standard qualitative techniqueds [27]. These observations were made as part of ongoing operational research [28] to understand and improve the services of the clinic. Key program components and issues were identified and are described in the results section below.

\section{Results}

\subsection{Existing Networks of CWHs}

CHWs were already an established part of the health system in Lesotho at the time the RHI started. Each village had one or two individuals - usually women-who were selected by the village chief to be a CHW. Prior to the RHI, these women had nebulous roles in their communities and were asked to be responsible for "the health of the village". They were not paid for any of their work nor were they assigned any specific tasks. They were not offered any ongoing training and received little to no support in the field. Many of them were too elderly to make visits to individuals living in their villages, and they themselves were often infected and sick with HIV. There was no infrastructure in which they could operate and no chain of command. Thus, although theoretically in existence, the CHWs were essentially defunct.

\subsection{Program Components}

\subsubsection{Selection of CHWs}

The RHI was committed to using CHWs in the scale-up of HIV care in the mountains of Lesotho. This decision was made based on the group's prior experience with successful HIV treatment programs in both Haiti and Rwanda [29]. The first task for the clinic team was to identify individuals who were willing to participate in the RHI program. The program planned to initiate treatment in at least 300 patients in the first 3 months of operations. Based on prior work, a ratio of $1 \mathrm{CHW}$ to 4 or 5 patients was felt to be optimal. In order to accommodate these patients and to allow for CHW drop out, $75 \mathrm{CHWs}$ was the target number for initial recruitment.

As noted in the section above, networks of CHWs already existed in the mountains of Lesotho, and it was from this pool of individuals that the original $75 \mathrm{CHWs}$ were selected. There were 34 villages surrounding Nohana Health Center, and the chiefs and CHWs from each village were approached by clinic staff and asked about their willingness to participate in the program. In some of the villages, the existing CHWs were either dead or did not express interest in the program. In these cases, the chief was asked to nominate an additional $\mathrm{CHW(s)}$ who were willing to participate. Most villages had two CHWs each, although some had one and others had three. Ninety percent of the CHWs were women. All of the CHWs could read and write.

\subsubsection{Initial Training}

An initial 5 day training program was conducted at Nohana health center in June of 2006. All 75 CHWs participated in the training. The curriculum was based on a successful HIV training course for CHWs developed in Haiti and then adapted to Rwanda [30]. Topics covered in the curriculum are listed in Table 1 . The curriculum was translated into the local language of Sesotho and cultural adaptations were made by a trained anthropologist. 
Table 1. Topics covered in initial training.

\begin{tabular}{|c|c|c|}
\hline Training Day & Unit & Topic \\
\hline \multirow[t]{4}{*}{ Day 1} & 1 & Introduction and overview of CHWs and HIV \\
\hline & 2 & HIV basic facts \\
\hline & 3 & Prevention and transmission of HIV \\
\hline & 4 & Treatment of HIV \\
\hline \multirow[t]{3}{*}{ Day 2} & 5 & Side effects of antiretroviral therapy \\
\hline & 6 & Women and HIV (including prevention of maternal to child transmission and family planning) \\
\hline & 7 & Other sexually transmitted diseases \\
\hline \multirow[t]{4}{*}{ Day 3} & 8 & Stigma and discrimination \\
\hline & 9 & Psychosocial support and effective communication \\
\hline & 10 & Tuberculosis \\
\hline & 11 & Tuberculosis treatment and side effects \\
\hline \multirow[t]{2}{*}{ Day 4} & 12 & Other opportunistic infections \\
\hline & 13 & Nutrition and HIV \\
\hline \multirow[t]{3}{*}{ Day 5} & 14 & Roles and responsibilities of CHWs \\
\hline & 15 & Confidentiality \\
\hline & 16 & Challenges faced by CHWs \\
\hline
\end{tabular}

Trainings were led by the RHI team and the Nohana Clinic Staff. Training was conducted in Sesotho or in English with a Sesotho translation. Each CHW was given a training manual and a CHW notebook for their work. During their training, the CHWS were shown slides relevant to the topics of discussion. Multiple case studies were discussed during the training in both small and large groups.

\subsubsection{Job Responsibilities}

CHWs were paid a per diem of 50 maluti per day (8 USD). They were also given two meals during the training. Any transport costs they incurred were reimbursed, and for those living far from the health center, overnight accommodations were provided. All 75 participants completed the 5 day training. Photos of the training are shown in Figures 1 and 2.

The CHWs were all given very clear job responsibilities and tasks for which they would now be responsible. The activities focused on four main areas: 1) daily contact with individuals on antiretroviral therapy to ensure mediations were being taken correctly and to assess for any clinical changes that would prompt a visit to the health center (i.e. signs of adverse effects, signs of possible new opportunistic infections, etc.); 2) accompanying all patients with HIV to scheduled clinic visits; 3) identification of individuals in the village who appear to be in need of medical care and either bring them to the clinic

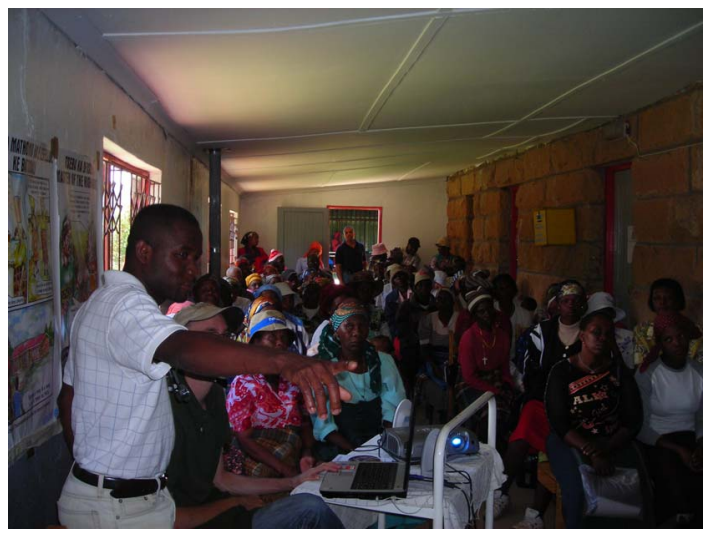

Figure 1. CHWs at nohana training.



Figure 2. CHWs at monthly meeting. 
for care, or if the person was too sick to get to the clinic, arrange for clinical staff to do a home visit; and 4) general community outreach and education about HIV and other health concerns. Each of these will be described in more detail.

1) Daily contact with individuals on antiretroviral therapy.

CHWs were responsible for making a daily visit to each person in their village who was on ART. The purpose of the home visit was to ensure that medications were being taken as prescribed and to answer any question the patient and his or her family might have about the medications. CHWs were trained about the basic antiretrovirals and were given a medication list for each patient in their village to ensure the patient had the correct medications. If there were discrepancies, the $\mathrm{CHW}$ went to the health center to verify the medication regimen. The daily visits also served as a way to assess the general health status of the patient. CHWs were trained to ask a series of basic screening questions regarding signs or symptoms that needed further assessment. If these symptoms were present, then the CHW notified the clinic for follow-up. In addition to these duties, the CHWs were asked to do the following at each daily visit: provide physical and emotional support for the patient and his or her family; identify other members of the household in need of clinical assessment; assess social and economic factors that might impact patients' health; and provide directly observed therapy for other medications (i.e. TB treatment).

2) Accompany all patients with HIV to scheduled health center appointments.

CHWs were assigned to accompany all HIV-positive individuals to their scheduled visits at the health center. The purpose of this was to ensure that patients were able to keep their scheduled appointments. Having both the CHW and the patient at the clinic appointment also ensured that the $\mathrm{CWH}$ and patient were given the same information on the patient's treatment plan for the coming months. Finally, the CHW was also asked to come to the visit to be an advocate for the patient and raise any concerns that the patient may not be comfortable voicing to providers. With regard to the health center appointments, the CHWs were asked to do the following: Remind patients of upcoming appointments at the clinic; work with the patient to make a list of topics to discuss at the appointment; escort patients to the clinic for their appointments; arrange for safe and reliable transport for patients not able to walk to clinic; record in their CHW notebook the treatment plan for each patient; discuss the list of patient concerns with the providers; document the date of the next appointment.

3) Identify individuals in the community in need of medical attention.
Most of the villages that utilized Nohana health center were located some distance from the clinic. On average, people had to walk two to three hours over rugged terrain to reach the clinic. At the start of the program, many of the patients with HIV were too ill to walk to the health center. Thus, CHWs were asked to identify persons in their villages who were ill and in need of medical attention. When possible, the clinics worked with the CHWs to arrange transportation to the clinic. In many cases, however, there were no means of transporting the patient to the clinic outside of carrying him or her. When this was the case, CHWs reported to the clinic and arranged for home visits to be made to the community members. During these home visits, the CHW came to the clinic and took the clinic staff (usually a physician or nurse) to the home or homes of those individuals in need of care. HIV testing and ART initiation was then done in the home for those in need of it. Patients could also undergo TB testing and TB treatment initiation in the home as well. Other basic medical assessments could be done at this time.

Home visits often served as a way to inform the community about the HIV and other programs at the health center. It was often the case that multiple other individuals in need of assessment and care were identified. When possible, these individuals were seen one the date of the original home visit. If more individuals were identified than could be seen, follow up visits were schedule. This also included general community screenings for HIV, malnutrition, vaccinations, and other community needs.

With regards to their work in identifying individuals in the community in need of medical attention, CHWs were specifically asked to: seek out individuals and families who were felt to be "sick"; approach these individuals and their families and ask if they would like a home visit from clinic staff; report these individuals to the clinic staff and plan for a transport or a home visit; accompany clinic staff to the home or homes of identified individuals; arrange for any follow-up needed for those seen on home visits; schedule additional home visits for patients not able to be seen at the original home visit; identify at-risk groups in need of community screening for HIV, TB, nutrition, and other health issues.

4) Provide general outreach and education to the community.

CHWs were not only responsible to individuals with identified health needs, but also to the community as a whole. To this end, they were responsible for providing the community with general education about HIV and AIDS, including risk factors, transmission, prevention, testing and treatment. CHWs were also responsible to provide the community with education about other important health topics. Some of these topics were identified by the Nohana clinic staff as affecting the region (i.e. 
vaccination campaigns, food shortages, etc.). Others were identified by the community and the CHWs worked with the clinic staff to develop programs in these areas (i.e. male circumcision, migrant labor and HIV, working with traditional healers). More specifically, CHWs were asked to: organize community workshops on HIV; organize community HIV testing drives; advise community members about clinic services; provide condoms for HIV prevention; survey the community about pressing health concerns; organize workshops for the community on other health topics.

\subsubsection{Payment}

All CHWs were provided with a monthly payment for their work, the rate of which was determined by the MOHSW at 100 Maulti per CWH per month (USD 15). Payment was felt to improve the ability of the CHWs to perform their jobs and demonstrate the importance of the work they did. Many of the CHWs were themselves facing poverty and sickness, and it was felt that asking them to "volunteer" to do such important work would be unsustainable. Although this was an initial up-front cost to the program, it was felt that the benefits of keeping patients adherent and in treatment outweighed these costs. After a year of successful implementation and payment of the CWHs, the MOHSW of Lesotho decided to begin paying all the CHWs in the country.

\subsubsection{Ongoing Training}

In addition to the initial broad training session, ongoing education was offered in the form of monthly training sessions. These trainings not only provided ongoing educational material but also served to keep the CHWs connected to the RHI and health facility staffs, update the CHWs on information related to patients, diseases, and program developments, and allow the CHWs a regular forum to provide feedback. Regular trainings also reinforced the professionalization of the CHW's roles. The ongoing monthly trainings lasted between three and four hours, and a meal was provided. Transportation costs to attend the meeting were also subsidized.

In addition to ongoing training for existing CHWs, cadres of new CHWs were needed to be recruited and trained to meet expanding program needs. Given the expansion of clinical services in Nohana, an additional 65 CHWs needed to be trained and deployed in the field. Additional CHWs were selected from persons either nominated by the village chief or nominated by an active CHW. These new CHWs then underwent the initial 5 days training, which was held quarterly for new recruits. These CHWs then joined the ongoing training session and worked closely with a more experienced CHW during their first three months in the field.

\subsubsection{Ongoing Support and Supervision}

CHWs expressed the need for ongoing support from the health centers. Some of this was done at the monthly meetings discussed above. In addition, health center personnel also accompanied the CHWs into their communities and on home visits. Clinic staff provided ongoing support and feedback to CHWs and also gave them formal quarterly evaluations. Any problems that arose were discussed with suggestions for improvements. CHWs were relieved of their duties if they violated the privacy or confidentiality of a patient or if they knowingly committed acts that jeopardized patients' health.

CHWs themselves were supervised by the health center staff but also by the patients they cared for and the village as a whole. If there was a problem with a CHW, the clinic staff was notified, and a meeting was held with the clinic medical director and the CHW. Identification and mitigation of problems was the goal of the meeting, and most disputes could be solved. In settings where CHWs violated patient confidentiality or did not provide the patient with necessary medicines, that worker could be terminated.

In addition to this support and supervision, newly recruited and trained CHWs were matched with more experienced CHWs during their first three months in the field. This support was not available for the initial 75 CHWs, and their supervision and support was provided largely by clinic staff. Once this initial cadre gained field experience, they took over the majority of supervision and support of newly recruited CHWs. This provided more frequent interactions with supervisors who had direct experience with the responsibilities of the CHW and could offer practical, field-based advice. Quarterly supervision and feedback was given to all CHWs by the clinic staff.

\subsection{Challenges}

There were several challenges faced by the CHWs and in the implementation of the CHW program in Lesotho. Chief among these was stigma. Many patients expressed a fear of having CHWs come to their homes. They felt this might label them as having HIV. They also worried that someone in their village might "gossip" about them or share their status with others in the community.

This problem was managed by acknowledgement of the potential for problems and a frank discussion with CHWs and HIV patients. CHWs were trained in the principles and application of confidentiality and privacy on an ongoing basis. Those found to violate these principles received strict disciplinary action. In addition, once patients were receiving treatment, a majority of them got better, and stigma was lessened overall. The CHWs were seen to play an important role in this, and they became 
welcome visitors in the village among those with HIV.

A second problem was the distance most CHWs lived from the clinic. Many had to walk 2 - 3 hours over difficult terrain to reach the health center. This was a problem if they identified a critically ill patient in need of emergent care. This was also a problem if they were supporting several different patients who came to clinic on different days. At the beginning of the program CHWs who supported patients in other villages that are several hours away led to neglect of patients

In order to address these issues, CHWs were given transportation stipends. Efforts were made by the clinic to schedule all the follow-up patients for one CHW on the same day. Perhaps most importantly, new CHWs were recruited and trained from all the villages, thus leading to a situation in which most CHWs were in close proximity to the patients they supported.

\section{Conclusions}

CHWs were crucial scaling up HIV treatment in rural Lesotho, much as they have been in other programs throughout the world. This paper discusses the practical implementation of CHW program at Nohana clinic in rural Lesotho, a resource-poor, high burden HIV setting. CHWs were successful in starting and marinating thousands of individuals on HIV therapy in this setting. They were carefully selected from pools of existing CHWs which had floundered in the absence of training and support. They were largely female and could read and write. They received initial and ongoing training for HIV-related work using a curriculum developed in other settings and adapted to Lesotho. They carried out a number of activities, including community education and mobilization, adherence support, and active case finding. They were paid for their work and supported by health center staff at the clinic. The CHWs in Lesotho were highly motivated and successful at what they did, and it is hoped their experience can be a model for other CHW programs. In addition to their HIV work, CHWs could also be successfully trained to manage other chronic health problems, sustain existing communicable disease prevention and treatment programs, and participate in community mobilization and outreach on a number of health issues.

There are multiple limitations to this paper. First, the data reported is based on field observations conducted over a 30 month time period in a single clinic. There may be both historical and reporting bias inherent in these results. In addition, this data is not generalizable to the general population. Finally, the purpose of this research was to describe the process of CHW selection, training, and supervision and is not linked to outcomes at the patient or clinic level.
In spite of these limitations, this paper offers important insight into the CHW program used to roll-out HIV prevention and treatment services in the mountains of Lesotho. While there is much written about CHW programs, there is little in the literature about the selection, training, payment, supervision, and specific responsibilities of this group of health paraprofessionals. This paper can assist programs wanting to deploy CHWs define and carry out the steps that are needed to ensure these workers can be effective in the field of HIV care. Given the high burden of HIV disease in the very settings where HIV is rampant, such operational guidance is necessary to ensure that HIV care is available to all.

\section{Acknowledgements}

The authors of this paper are most thankful to and mindful of the men and women of Lesotho who inspire us with their daily courage in the fight against HIV. We are also thankful to all the clinic staff who provided daily care to those living with HIV and AIDS. For program support work we acknowledge and are thankful to Jaclyn Chai, Bob Hsiung, Cheryl Snyder, Ted Constan, Jeremy Keeton, Hind Satti, and Kwonjune Seung. This work would not have been possible without the Ministry of Health and Social Welfare of Lesotho and our other implementing partners, including the Clinton HIV/AIDS Initiative, Mission Aviation Fellowship, and Catholic Relief Services. For funding support, we thank Irish Aid, the Francois Xavier Bagnoud Center for Health and $\mathrm{Hu}-$ man Rights at the Harvard School of Public Health, Partners in Health, Thomas J. White, and Frank Hatch.

\section{REFERENCES}

[1] H. Standing and A. M. Chowdhury, "Producing Effective Knowledge Agents in a Pluralistic Environment: What Future for Community Health Workers?” Social Science \& Medicine, Vol. 66, No. 10, 2008, pp. 2096-2107. doi:10.1016/j.socscimed.2008.01.046

[2] B. Devkota and E. van Teijlingen, "Demystifying the Maoist Barefoot Doctors of Nepal,” Medicine, Conflict \& Survival, Vol. 26, No. 2, 2010, pp. 108-123. doi:10.1080/13623699.2010.491382

[3] L. M. Perez and J. Martinez, "Community Health Workers: Social Justice and Policy Advocates for Community Health and Well-Being," American Journal of Public Health, Vol. 98, No. 1, 2008, pp. 11-14. doi:10.2105/AJPH.2006.100842

[4] A. Haines, D. Sanders, U. Lehmann, A. K. Rowe, J. E. Lawn, S. Jan, D. G. Walker and Z. Bhutta, “Achieving Child Survival Goals: Potential Contribution of Community Health Workers,” Lancet, Vol. 369, No. 9579, 2007, pp. 2121-2131. doi:10.1016/S0140-6736(07)60325-0

[5] S. A. Lewin, J. Dick, P. Pond, M. Zwarenstein, G. Aja, B. van Wyk, X. Bosch-Capblanch and M. Patrick, "Lay 
Health Workers in Primary and Community Health Care,” Cochrane Database of Systematic Reviews, Vol. 1, 2005, Article ID: CD004015. doi:10.1002/14651858.CD004015.pub2

[6] M. A. Nemcek and R. Sabatier, "State of Evaluation: Community Health Workers," Public Health Nursing, Vol. 20, No. 4, 2003, pp. 260-270. doi:10.1046/j.1525-1446.2003.20403.x

[7] J. M. Stacciarini, A. Rosa, M. Ortiz, D. B. Munari, G. Uicab and M. Balam, "Promotoras in Mental Health: A Review of English, Spanish, and Portuguese Literature,” Family \& Community Health, Vol. 35, No. 2, 2012, pp. 92-102.

[8] C. Giugliani, E. Harzheim, M. S. Duncan and B. B. Duncan, "Effectiveness of Community Health Workers in Brazil: A Systematic Review," Journal of Ambulatory Care Management, Vol. 34, No. 4, 2011, pp. 326-338.

[9] L. Ruggiero, A. Castillo, L. Quinn and M. Hochwert, "Translation of the Diabetes Prevention Program's LifeStyle Intervention: Role of Community Health Workers," Current Diabetes Reports, Vol. 12, No. 2, 2012, pp. 127-137. doi:10.1007/s11892-012-0254-y

[10] C. F. Reynolds III, P. Cuijpers, V. Patel, A. Cohen, A. Dias, N. Chowdhary, O. I. Okereke, M. A. Dew, S. J. Anderson, S. Mazumdar, F. Lotrich and S. M. Albert, "Early Intervention to Reduce the Global Health and Economic Burden of Major Depression in Older Adults," Annual Review of Public Health, Vol. 33, 2012, pp. 123135. doi:10.1146/annurev-publhealth-031811-124544

[11] M. E. Herce, J. A. Chapman, A. Castro, G. Garcia-Salyano and K. Khoshnood, "A Role for Community Health Promoters in Tuberculosis Control in the State of Chiapas, Mexico,” Journal of Community Health, Vol. 35, No. 2, 2010, pp. 182-189. doi:10.1007/s10900-009-9206-0

[12] D. G. Datiko and B. Lindtjorn, "Cost and Cost-Effectiveness of Smear-Positive Tuberculosis Treatment by Health Extension Workers in Southern Ethiopia: A Community Randomized Trial,” PLoS One, Vol. 5, No. 2, 2010, Article ID: e9158. doi:10.1371/journal.pone.0009158

[13] S. Alamo, F. Wabwire-Mangen, E. Kenneth, P. Sunday, M. Laga and R. L. Colebunders, "Task-Shifting to Community Health Workers: Evaluation of the Performance of a Peer-Led Model in an Antiretroviral Program in Uganda," AIDS Patient Care and STDs, Vol. 26, No. 2, 2012, pp. 101-107. doi:10.1089/apc.2011.0279

[14] S. Kenya, N. Chida, S. Symes and G. Shor-Posner, "Can Community Health Workers Improve Adherence to Highly Active Antiretroviral Therapy in the USA? A Review of the Literature," HIV Medicine, Vol. 12, No. 9, 2011, pp. 525-534. doi:10.1111/j.1468-1293.2011.00921.x

[15] L. C. Ivers, J. G. Jerome, K. A. Cullen, W. Lambert, F. Celletti and B. Samb, "Task-Shifting in HIV Care: A Case Study of Nurse-Centered Community-Based Care in Rural Haiti,” PLoS One, Vol. 6, No. 5, 2011, Article ID: e19276. doi:10.1371/journal.pone.0019276

[16] N. van Ginneken, S. Lewin and V. Berridge, “The Emergence of Community Health Worker Programmes in the
Late Apartheid Era in South Africa: An Historical Analysis,” Social Science \& Medicine, Vol. 71, No. 6, 2010, pp. 1110-1118. doi:10.1016/j.socscimed.2010.06.009

[17] E. Wouters, W. Van Damme, D. van Rensburg, H. Meulemans, "Impact of Baseline Health and Community Support on Antiretroviral Treatment Outcomes in HIV Patients in South Africa,” AIDS, Vol. 22, No. 18, 2008, pp. 2545-2548. doi:10.1097/QAD.0b013e32831c5562

[18] N. Ford, K. Kranzer, K. Hilderbrand, G. Jouquet, E. Goemaere, N. Vlahakis, L. Trivino, L. Makakole and H. Bygrave, "Early Initiation of Antiretroviral Therapy and Associated Reduction in Mortality, Morbidity and Defaulting in a Nurse-Managed, Community Cohort in Lesotho,” AIDS, Vol. 24, No. 17, 2010, pp. 2645-2650. doi:10.1097/QAD.0b013e32833ec5b2

[19] E. Gouws, K. A. Stanecki, R. Lyerla and P. D. Ghys, "The Epidemiology of HIV Infection among Young People Aged 15 - 24 Years in Southern Africa,” AIDS, Vol. 22, No. S4, 2008, pp. S5-S16. doi:10.1097/01.aids.0000341773.86500.9d

[20] J. J. Furin, H. L. Behforouz, S. S. Shin, J. S. Mukherjee, J. Bayona, P. E. Farmer, J. Y. Kim and S. Keshavjee, "Expanding Global HIV Treatment: Case Studies from the Field,” Annals of the New York Academy of Sciences, Vol. 1136, 2008, pp. 12-20. doi:10.1196/annals.1425.004

[21] J. K. Joseph, J. Rigodon, C. Cancedda, M. Haidar, N. Lesia, L. Ramanagoela and J. Furin, "Lay Health Workers and HIV Care in Rural Lesotho: A Report from the Field,” AIDS Patient Care \& STDs, Vol. 26, No. 3, 2012, pp. 141-147. doi:10.1089/apc.2011.0209

[22] J. J. Furin, J. Rigodon, C. Cancedda, S. Keshavjee, K. J. Seung, M. Letsie, J. Y. Kim and J. K. Joseph, "Improved Case Detection of Active Tuberculosis Associated with an Antiretroviral Treatment Program in Lesotho," International Journal of Tuberculosis \& Lung Disease, Vol. 11, No. 10, 2007, pp. 1154-1156.

[23] J. Furin, M. Shutts and S. Keshavjee, “Aviation and the Delivery of Medical Care in Remote Regions: The Lesotho HIV Experience,” Aviation Space \& Environmental Medicine, Vol. 79, No. 2, 2008, pp. 136-138. doi:10.3357/ASEM.2134.2008

[24] J. Furin, "The Role of Traditional Healers in CommunityBased HIV Care in Rural Lesotho,” Journal of Community Health, Vol. 36, No. 5, 2011, pp. 849-856. doi:10.1007/s10900-011-9385-3

[25] M. L. Rich, A. C. Miller, P. Niyigena, M. F. Franke, J. B. Niyonzima, A. Socci, P. C. Drobac, M. Hakizamungu, A. Mayfield, R. Ruhayisha, H. Epino, S. Stulac, C. Cancedda, A. Karamaga, S. Niyonzima, C. Yarbrough, J. Fleming, C. Amoroso, J. Mukherjee, M. Murray, P. Farmer and A. Binagwaho, "Excellent Clinical Outcomes and High Retention in Care among Adults in a Community-Based HIV Treatment Program in Rural Rwanda,” Journal of Acquired Immune Deficiency Syndromes, Vol. 59, No. 3, 2012, pp. e35-e42. doi:10.1097/QAI.0b013e31824476c4

[26] L. C. Ivers, J. S. Mukherjee, F. R. Leandre, J. Rigodon, K. A. Cullen and J. Furin, "South-South Collaboration in Scale-Up of HIV Care: Building Human Capacity for 
Care,” AIDS, Vol. 24, Suppl. 1, 2010, pp. S73-S78. doi:10.1097/01.aids.0000366085.14064.6f

[27] R. G. Jones, A. N. Trivedi and J. Z. Ayanian, "Factors Influencing the Effectiveness of Interventions to Reduce Racial and Ethnic Disparities in Health Care," Social Science \& Medicine, Vol. 70, No. 3, 2011, pp. 337-341. doi:10.1016/j.socscimed.2009.10.030

[28] A. D. Harries, I. D. Rusen, T. Reid, A. K. Detjen, S. D. Berger, K. Bissell, S. G. Hinderaker, M. Edginton, M. Fussell, P. I. Fujiwara and R. Zachariah, "The Union and Medecins Sans Frontieres Approach to Operational Re- search," International Journal of Tuberculosis \& Lung Disease, Vol. 15, No. 2, 2011, pp. 144-154.

[29] M. M. Alsan, M. Westerhaus, M. Herce, K. Nakashima and P. E. Farmer, "Poverty, Global Health, and Infectious Disease: Lessons from Haiti and Rwanda," Infectious Disease Clinics of North America, Vol. 25, No. 3, 2011, pp. 611-622. doi:10.1016/j.idc.2011.05.004

[30] Partners in Health, “Accompagnateurs Curriculum," Boston, 2012.

http://www.pih.org/publications/entry/accompagnateurs-c urriculum 